\title{
Exploring the application of copy number variation sequencing in prenatal diagnosis
}

\author{
Tianyuan Zhang ${ }^{1}$, Suzhen $\mathrm{Qu}^{2}$, Zhi $\mathrm{Gao}^{1}$, Panlai $\mathrm{Shi}^{1}$, and Xiangdong Kong ${ }^{3}$ \\ ${ }^{1}$ Zhengzhou University First Affiliated Hospital \\ ${ }^{2}$ xin yang shi zhong xin yi yuan \\ ${ }^{3}$ Affiliation not available
}

September 16, 2020

\begin{abstract}
Objective: To explore whether CNV-seq can be used as a first-tier diagnostic method or even replace karyotyping for prenatal diagnosis alone. Method: A retrospective study was conducted to 4230 amniocentesis samples with CNV-seq detection and G-banding karyotyping simultaneously. The indications for prenatal diagnosis included abnormal result on Down's Syndrome Screening, abnormal fetal ultrasound, abnormal result on noninvasive prenatal screening, and so on. The results that lead to birth defects definitely were defined as abnormalities, which included aneuploidy, mosaic aneuploidy, large deletion/duplication and pathogenic copy number variations (pCNVs). Results: 278 cases of abnormalities was identified by karyotyping with an abnormal detection rate of $6.69 \%(283 / 4230)$. In addition, for all the abnormalities identified by karyotyping, CNV-seq also identified another 58 cases of abnormalities. A total of 341 cases of abnormalities were identified by CNV-seq with an abnormal detection rate of $8.06 \%(341 / 4230)$, higher than that of karyotyping. Abnormal detection rate of CNV-seq for the groups with abnormal result on noninvasive prenatal screening, abnormal fetal ultrasound, abnormal parental chromosome, adverse pregnancy history, abnormal result on Down's Syndrome Screening, volunory testing and advanced maternal age were increased by $2.53 \%, 1.91 \%, 1.44 \%, 1.24 \%, 1.01 \%, 0.99 \%$ and $0.62 \%$ over the karyotyping respectively. Conclusion: CNV-seq and karyotyping had the same effectiveness in identifying aneuploidies, but CNV-seq had absolute superiority in the detection of low proportion of mosaics, imbalanced structural abnormalities. This prevents the birth of fetuses with these chromosome abnormalities that cannot be identified by karyotyping. CNV-seq can replace karyotyping in prenatal diagnosis for chromosome test alone.
\end{abstract}

\section{Tweetable abstract}

CNV-seq can replace karyotyping in prenatal diagnosis for chromosome test alone.

Funding: No funding support.

Keywords: copy number variation sequencing (CNV-seq), G-banding karyotyping, prenatal diagnosis, aneuploidy, copy number variations (CNVs), variants of uncertain significance (VOUS).

\section{INTRODUCTION:}

In recent years, copy number variation sequencing (CNV-seq) has been gradually applied in prenatal diagnosis. Is this technique used alone or in combination with karyotyping in prenatal diagnosis? This issue is confusing for clinicians.

Aneuploidy, large deletions/duplications and pathogenic copy number variations(pCNVs) are the major causes of birth defects ${ }^{[1]}$. Karyotyping has been the "gold standard" of chromosome test. It can identify 
the majority of chromosome abnormalities, which included polyploidy, aneuploidy, greater than $10 \mathrm{Mb}$ deletion/duplication, balanced structure rearrangement (reciprocal translocation, Robertsonian translocation, inversion, etc.). However, it has long detection period, low success rate, low resolution (chromosome abnormality less than $5 \mathrm{Mb}$ even $10 \mathrm{Mb}$ cannot be identified) ${ }^{[2]}$. Chromosomal microarray analysis (CMA) can provide higher resolution. This can detect genome-wide imbalance chromosomal changes and pinpoint the location of abnormal changes. However, the cost of CMA was higher. The chromosomal regions that were not covered by probes cannot be detected ${ }^{[3]}$. Therefore, there is an urgent need for a more economical method to detect chromosomal disorders comprehensively and accurately. Copy number variation sequencing (CNV-seq) based on next generation sequencing(NGS) technology provides a new method for prenatal diagnosis. Compared with karyotyping, it has the advantages of wide detection range, high resolution, high success rate, easy operation and short detection period. This study retrospectively analyzed amniocentesis samples that were analyzed by both CNV-seq and karyotyping, aiming to explore the value of CNV-seq in prenatal diagnosis.

\section{Objects and Methods:}

\section{Research Objects:}

In this study, a retrospective study was conducted on 4158 pregnant women whose age ranged from 15 to 52 years old and gestational weeks ranged from $15^{+4}$ to $33^{+6}$. They underwent amniocentesis and chromosome test by CNV-seq and karyotyping simultaneously at the center of genetic and prenatal diagnosis of the first affiliated hospital of Zhengzhou University from May 2018 to December 2019. The indications for prenatal diagnosis included abnormal results on Down's Syndrome Screening(1889 cases), abnormal fetal ultrasound(680 cases), abnormal result on noninvasive prenatal screening(631 cases), advanced maternal age(480 cases), adverse pregnancy history(241 cases), abnormal parental chromosomes(208 cases), and voluntary testing(101 cases). This study was approved by the medical ethics committee of the first affiliated hospital of Zhengzhou University, and all patients signed the informed consent.

\section{Research method:}

1. Amniocentesis and karyotyping: Amniocentesis were conducted under ultrasonic guidance under aseptic conditions. $20 \mathrm{~mL}$ amniotic fluid was obtained. $15 \mathrm{ml}$ of it was used for $\mathrm{G}$ banding karyotyping, and $5 \mathrm{~mL}$ of it was used for CNV-seq detection. Genomic DNA of amniotic fluid cells was extracted by purified column method (QIAamp DNA Blood Mini Kit, QIAGEN company, Germany). Quantitative fluorescence polymerase chain reaction (QF-PCR) was used to exclude maternal cell contamination and polyploidy. When the maternal signal was more than 10\%, it indicated maternal cell contamination.

2. G-banding Karyotyping: amniotic fluid cells were cultured and harvested after being stimulated with phytohemagglutinin for $72 \mathrm{~h}$. Metaphase chromosomes were prepared according to the standard cytogenetic protocols ${ }^{[4]}$.

3. CNV-seq detection: The commercial CNV detection library construction kit (Berry Genomics corporation, Beijing) was used. The CNVs were detected by Illumina NextSeq 500. Their sequencing type is SE45 (single ended sequencing, read length: 45bp) and average sequencing depth is $0.1 \mathrm{x}$. Human genome reference sequence version GRCh37 (UCSC database, http://genome.ucsc.edu/cgibin/hgGateway) was selected. Tattini et al ${ }^{[5]}$ analyzed sequencing data by the CNV detection algorithm. The resolution of CNVs was more than 100kb. The main reference for pathogenicity analysis of CNVs is the Database of Genomic Variants (DGV, http://dgv.tcag.ca/dgv/app/home), DECIPHER (https://decipher.sanger.ac.uk), OMIM (https://omim.org), PubMed (https://www.ncbi.nlm.nih.gov/pubmed/) and ClinGen (https://www.ncbi.nlm.nih.gov/projects/dbvar/clingen). CNVs is divided into pCNVs, benign CNVs(bCNVs) and variants of uncertain significance(VOUS). CNVs classification was performed based on the guidelines of the American College of Medical Genetics ${ }^{[6]}$.

\section{Results:}

First: summary and comparison of the results of karyotyping and CNV-seq. 
4142 cases of 4230 were analyzed by karyotyping successfully (88 cases failed), with a success rate of $97.92 \%$. 4229 cases were successfully analyzed by CNV-seq (1 case failed), with a success rate of $99.98 \%$. Karyotyping results included 235 cases of aneuploidies, 18 cases of mosaic aneuploidies, 30 cases of large deletions/duplications, 133 cases of balanced rearrangements, 88 cases of suspicious structure changes, 94 cases of polymorphism and 3544 cases of normal. CNV-seq results included 240 cases of aneuploidies, 22 cases of mosaic aneuploidies, 79 cases of pCNVs, 93 cases of VOUS, 102 cases of bCNVs and 3693 cases of normal. The results that lead to birth defects are defined as abnormalities. They included aneuploidy, mosaic aneuploidy, large deletions/duplications and pCNVs. The summary and comparison of all karyotyping and $\mathrm{CNV}$-seq results are shown in table 1.

A total of 283 cases of abnormalities were identified by karyotyping and CNV-seq simultaneously(table 1 red), which included 235 cases of aneuploidies, 18 cases of mosaic aneuploidies, 30 cases of large deletions/duplications or pCNVs. A total of 58 cases of abnormalities were identified by CNV-seq alone(table 1 green), which included 5 cases of aneuploidies, 4 cases of mosaic aneuploidies and 49 cases of pCNVs. Aneuploidies and mosaic aneuploidies were identified by Karyotyping and CNV-seq simultaneously (table 2): 131 cases of trisomy 21(including 2 cases of mosaics), 39 cases of trisomy18, 26 cases of 47,XXY (including one mosaic), 19 cases of monosomy X (including 11 cases of mosaics), 18 cases of 47, XXX, 14 cases of 47,XYY, 3 cases of trisomy 13 (including 2 cases of mosaics), 1 case of mosaic trisomy 22,1 case of mosaic trisomy 15, 1 case of 48,XXYY. Aneuploidies and mosaic aneuploidies were identified by CNV-seq alone (table 2): 5 cases of trisomy 21(including 1 case of mosaic, mos0.25), 1 case of 47,XXX, 1 case of mosaic 47,XXY (mos0.2) (normal karyotype), 1 case of mosaic trisomy 7 (mos0.1) (normal karyotype), and 1 case of mosaic trisomy 9 (mos0.3) (normal karyotype).

79 cases of large deletions/duplicaitons or pCNVs are shown in table S1. Cases 1-30 with abnormalities were identified by CNV-seq and karyotyping simultaneously(a total of 30 cases). Cases 31-79 with abnormalities were identified by CNV-seq alone (a total of 49 cases). The karyotyping results of 79 cases with CNV-seq results of pCVNs were large deletion/duplication (30 case, case $\left.1^{\sim} 30\right)$, suspicious change(13 cases, case $\left.31^{\sim} 43\right)$, polymorphism(1 cases, case 44$)$, balanced structural change $\left(2\right.$ cases, case $\left.45^{\sim} 46\right)$ and normal $(33$ cases, case $47^{\sim} 79$ ). Among 79 cases of pCNVs, there were 4 cases of monogenic disease (steroid sulphatase deficiency) and 43 cases of identified chromosome disease syndrome. This included 9 cases of 22q11 duplication syndrome, 7 cases of 22q11 deletion syndrome (DiGeorge syndrome), 3 cases of $7 \mathrm{q} 11.23$ duplication syndrome, 2 cases of 2q37monosomy syndrome, 2 cases of Cri du Chat Syndrome (5p deletion), 2 cases of Williams-Beuren Syndrome, 2 cases of 8p23.1 deletion syndrome, 2 cases of $18 \mathrm{p}$ deletion syndrome, and all other cases of 14 types of chromosome disease syndromes. The summary and comparison of all structural abnormalities are shown in Table 3.

Table S2 showed 102 cases of bCNVs(case 1 102), 93 cases of VOUS(case 103 195) of CNV-seq and corresponding karyotype results. In the samples with CNV-seq results of bCNVs, the karyotyping results were the suspicious change ( 1 case, case 1$)$, normal (89 cases, case $\left.2^{\sim} 90\right)$, polymorphism (5 cases, case $\left.91^{\sim} 95\right)$, balanced structural change ( 7 cases, case $\left.96^{\sim} 102\right)$. In the samples with CNV-seq results of VOUS, the karyotyping results were the balanced structural change( 6 cases, case $\left.103^{\sim} 108\right)$, suspicious change (4 cases, case 109 112), normal(79 cases, case 113 191), failed(4 cases, case 192 195). In this study, $14.39 \%$ (20/139) of the balanced rearrangements accompanied by unbalanced structural changes were identified by CNV-seq, including 7 cases of pCNVs (cases $39^{\sim} 45$ in table S1), 7 cases of bCNVs (cases $96^{\sim} 102$ in table S2) and 6 VOUS (cases $103^{\sim} 108$ in table S1).

\section{Second: comparison of CNV-seq and karyotyping in different clinical indication groups.}

According to indications for prenatal diagnosis, all cases were divided into groups of AR-DSS (1889 cases), AFU(680 cases), AR-NIPS(631 cases), AMA (480 cases), APH (241 cases), APC(208 cases) and VT (101 cases). The comparison of abnormal detection rates of two detection methods for all cases and groups are shown in figure 1.

\section{Discussions}


CNV-seq and karyotyping have the same effectiveness in aneuploidy detection, as shown in table 1. However, the abnormal detection rate of CNV-seq was higher than that of karyotyping ${ }^{[7]}$ in mosaic aneuploidy and imbalanced chromosome abnormalities. In addition, it has a higher detection success rate. Huilin Wang et $\mathrm{al}^{[8]}$ showed that CNV-seq needs lesser DNA and lower detection cost than CMA technology. We can identify additional and clinically significant CNVs with enhanced resolution and increased sensitivity of detecting mosaicism. The reason is that CMA has limited the coverage area of probe and cannot detect low proportion of mosaic. Compared with CMA, CNV-seq cannot detect the absence of heterozygosity (AOH). The absence of triploidy and polyploidy can be detected by CMA and karyotyping, but not by CNV-seq. Compared with karyotyping, CNV-seq cannot detect the absence of balanced rearrangement, such as reciprocal translocation, Robertsonian translocation, inversion, etc. In fact, most bases are in the state of AOH. Therefore, AOH is not pathogenic, unless there is a recessive homozygous mutation behind it, or a uniparental disomy with imprinted genes ${ }^{[9]}$. In addition, triploidy, polyploidy and "truly" balanced chromosomal rearrangements do not lead to birth defects (triploid and polyploid fetuses generally die in early pregnancy). It still requires further examination by CNV-seq for the cases with suspicious chromosome change, and the cases that have not been identified by karyotyping. Therefore, CNV-seq can completely replace the karyotyping in prenatal diagnosis for fetal chromosome analysis alone, as shown in figure 1.

With the improvement of the resolution by CNV-seq, some CNVs with variants of uncertain significance (VOUS), and even the unprecedented CNVs identified by CNV-seq will cause problems to clinicians' genetic counseling and pregnant women. This can cause unnecessary pregnancy termination. In this study, cases 6392 of CNVs classified as VOUS (case 63 classified as pCNVs) were reclassified as bCNVs, as shown in table S2. The reason is that these CNVs in fetuses were found to be inherited from one parent after parental verification. Thus, this technology can help us to avoid this trouble. The pathogenicity of some VOUS can be determined in the future with extensive application of CNV-seq and accumulation of databases, thus more pCNVs can be diagnosed in the future. CNV-seq is beneficial to the clinic with its extensive application.

In this study, abnormal results on Down's Syndrome Screening were the most cause of indications for amniocentesis, followed by abnormal fetal ultrasound, abnormal result on noninvasive prenatal diagnosis, then advanced maternal age, adverse pregnancy history, abnormal parental chromosomes, and voluntary testing. In fig.1, the abnormal rate of chromosome abnormality on the noninvasive prenatal screening group is the highest, followed by abnormal fetal ultrasound group and abnormal parental chromosomes group, then advanced maternal age group. In addition, the abnormal result on Down's Syndrome Screening group, adverse pregnancy history group and voluntary testing group are the lowest. In fig.1, abnormal detection rate of CNV-seq was higher than that of karyotyping, either in all cases or in each clinical indication groups. This value is especially high in the abnormal parental chromosomes group. Parental chromosome abnormalities are mainly the balanced rearrangement. Almost all the fetuses that can survive to the amniocentesis period were the same balanced rearrangement with their parents or normal karyotype. In addition, "true" balanced chromosome rearrangement do not cause fetal birth defects ${ }^{[10]}$. Some apparently balanced chromosome rearrangement may be accompanied by microdeletion/microduplication. This can be identified by CNV-seq (case $45^{\sim} 46$ in table S1 and case $96^{\sim} 108$ in table S2), even some of them were pCNVs (case $45^{\sim} 46$ in table S1). In this study, $1.44 \%$ of fetal chromosome abnormalities identified by CNV-seq in abnormal parental chromosomes group were all pCNVs. This may be missed by karyotying. Therefore, CNV-seq can be used for prenatal diagnosis in group of abnormal parental chromosomes. An inherited balanced rearrangement will have no consequences for the pregnancy, but is relevant to future reproductive counseling ${ }^{[11]}$. Therefore, peripheral blood karyotyping should be performed after birth on fetuses. Their parental balanced rearrangement will cause abnormal fetal chromosome for future fertility guidance.

\section{Disclosure of interests:}

The authors declare no competing interests on relevant financial, personal, political, intellectual or religious interests.

\section{Contribution to authorship:}


In this article, Kong and Qu are responsible for the construction of the idea of the article, and Qu also writes this article. Zhang is responsible for data collection and analysis, Shi is responsible for reports analysis, and Gao is responsible for data processing.

\section{Details of ethics approval:}

Ethics Committee of the first affiliated Hospital of Zhengzhou University. This article is a retrospective analysis of the data, does not involve or disclose the privacy of patients, and does not require informed consent.

\section{Funding:}

No funding support.

\section{References}

1. Evans MI, Wapner RJ, Berkowitz RL. Noninvasive prenatal screening or advanced diagnostic testing:caveat emptor[J]. Am J Obstet Gynecol, 2016;215:298-305. DOI: 10.1016/j.ajog.2016.04.029.

2. Shearer BM, Thorland EC, Carlson AW, et al. Reflex fluorescent in situ hybridization testing for unsuccessful product of conception cultures: a retrospective analysis of 5555 samples attempted by conventional cytogenetics and fluorescent in situ hybridization[J]. Genet Med. 2011;13:545-552.

3. Hayes JL, Tzika A, Thygesen H, et a1. Diagnosis of copy number variation by Illumina next generation sequencing is comparable in performance to oligonucleotide array comparative genomic hybridization[J]. Genomics, 2013;102:174-181. DOI: 10.1016/j.ygeno.2013.04.006.Dong Z, Zhang J, Hu P, et a1. Low-pass whole-genome sequencing in clinical cytogenetics: a validated approach $[J]$. Genet Med, 2016;18:940-948. DOI: 10.1038/gim.2015.199.

4. Shen J, Wu W, Gao C, et al. Chromosomal copy number analysis on chorionic villus samples from early spontaneous miscarriages by high throughput genetic technology[J]. Mol Cytogenet. 2016;9:7. DOI: $10.1186 / \mathrm{s} 13039-015-0210-\mathrm{z}$.

5. Tattini L, D' Aurizio R, Magi A. Detection of genomic structural variants from next-generation sequencing data[J]. Front Bioeng Biotechnol, 2015;3:92. DOI: 10.3389/fbioe.2015.00092.

6. Richards S, Aziz N, Bale S, et al. Standards and guidelines for the interpretation of sequence variants: a joint consensus recommendation of the American College of Medical Genetics and Genomics and the Association for Molecular Pathology[J]. Genet Med 2015;17:405-24. DOI: 10.1038/gim.2015.30.

7. Wang J, Chen L, Zhou C, et a1. Prospective chromosome analysis of 3429 amniocentesis samples in China using copy number variation sequencing[J]. Am J Obstet Gynecol, 2018;1:287.e1-287.e18. DOI: 10.1016/j.ajog.2018.05.030.

8. Wang H, Dong Z, Zhang R, et al. Low-pass Genome Sequencing Versus Chromosomal Microarray Analysis: Implementation in Prenatal Diagnosis[J]. Genet Med, 2020;22:500-510. DOI: 10.1038/s41436019-0634-7.

9. Riggs ER, Andersen EF, Cherry AM, et al. Technical Standards for the Interpretation and Reporting of Constitutional Copy-Number Variants: A Joint Consensus Recommendation of the American College of Medical Genetics and Genomics (ACMG) and the Clinical Genome Resource (ClinGen) [J]. 2020;22: 245-257. DOI: 10.1038/s41436-019-0686-8.

10. Rosenfeld JA, Tucker ME, Escobar LF, et al. Diagnostic utility of microarray testing in pregnancy loss[J]. Ultrasound Obstet Gynecol. 2015;46:478-86. DOI: 10.1002/uog.14866.

11. Wapner RJ, Martin CL, Levy B, et al. Chromosomal Microarray Versus Karyotyping for Prenatal Diagnosis[J]. 2012;367:2175-84. DOI: 10.1056/NEJMoa1203382.FiguresFIGURE 1 The comparison of abnormal detection rates of two detection methods for all cases and groups. Abbreviations: ARNIPS: abnormal results on noninvasive prenatal screening, AFU: abnormal fetal ultrasound, APC: abnormal parental chromosome, AMA: advanced maternal age, AR-DSS: abnormal result on down's syndrome screening, APH: adverse pregnancy history, VT: voluntary testing.TablesTABLE 1 The summary and comparison of all karyotyping and CNV-seq results. 


\begin{tabular}{|c|c|c|c|c|c|c|c|c|c|}
\hline $\begin{array}{l}\text { CNV- } \\
\text { seq }\end{array}$ & $\begin{array}{l}\text { CNV- } \\
\text { seq }\end{array}$ & $\begin{array}{l}\text { CNV- } \\
\text { seq } \\
\text { total }\end{array}$ & $\begin{array}{l}\text { CNV- } \\
\text { seq } \\
\text { aneuploidy }\end{array}$ & $\begin{array}{l}\text { CNV- } \\
\text { seq } \\
\text { ymosaic } \\
\text { aneu- } \\
\text { ploidy } \\
\text { ? }\end{array}$ & $\begin{array}{l}\text { CNV- } \\
\text { seq } \\
\text { pCNVs }\end{array}$ & $\begin{array}{l}\text { CNV- } \\
\text { seq } \\
\text { VOUS }\end{array}$ & $\begin{array}{l}\text { CNV- } \\
\text { seq } \\
\text { bCNVs }\end{array}$ & $\begin{array}{l}\text { CNV- } \\
\text { seq } \\
\text { normal }\end{array}$ & $\begin{array}{l}\text { CNV- } \\
\text { seq } \\
\text { failure }\end{array}$ \\
\hline \multicolumn{3}{|c|}{ karyotypingneuploidy235 } & 235 & & 15 & & 102 & 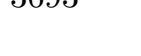 & \\
\hline & $\begin{array}{l}\text { mosaic } \\
\text { aneu- } \\
\text { ploidy }\end{array}$ & selication & & 18 & 30 & & & & \\
\hline & $\begin{array}{l}\text { balanced } \\
\text { rear- } \\
\text { range- } \\
\text { ment }\end{array}$ & 133 & & & 2 & 6 & 7 & 118 & \\
\hline & $\begin{array}{l}\text { suspicious } \\
\text { change }\end{array}$ & 88 & & & 13 & 4 & 2 & 69 & \\
\hline & polymorp & aistmm & & & 1 & & 3 & 90 & \\
\hline & normal & 3544 & & 3 & 33 & 79 & 90 & 3339 & \\
\hline & failure & 88 & 5 & 1 & & 4 & & 77 & 1 \\
\hline
\end{tabular}

NOTE: Red: abnormal results identified by karyotyping and CNV-seq simultaneously. Green: abnormal results identified by $\mathrm{CNV}$-seq alone.

TABLE 2 All results of aneuploidies and mosaic aneuploidies in 4230 samples.

\begin{tabular}{|c|c|c|}
\hline Aneuploidy & $\begin{array}{l}\text { Detected on karyotyping and CNV-seq simultaneously } \\
\text { Full }\end{array}$ & $\begin{array}{l}\text { Detected on karyotypin } \varepsilon \\
\text { Mosaics }\end{array}$ \\
\hline Any aneuploidy & 235 & 18 \\
\hline Common autosomal trisomy & 169 & 6 \\
\hline Trisomy 21 & 129 & 2 \\
\hline Trisomy 18 & 39 & 0 \\
\hline Trisomy 13 & 1 & 2 \\
\hline Trisomy 22 & 0 & 1 \\
\hline Trisomy 15 & 0 & 1 \\
\hline Trisomy 9 & 0 & 0 \\
\hline Trisomy 7 & 0 & 0 \\
\hline Sex chromosome aneuploidy & 66 & 12 \\
\hline $47, \mathrm{XXY}$ & 25 & 1 \\
\hline $45, \mathrm{X}$ & 8 & 11 \\
\hline $47, \mathrm{XXX}$ & 18 & 0 \\
\hline 47,XYY & 14 & 0 \\
\hline $48, X X Y Y$ & 1 & 0 \\
\hline
\end{tabular}

NOTE: Red: the normal karyotyping samples; Green: the failure karyotyping samples

TABLE 3 The summary and comparison of all phenotype of structural abnormalities.

Phenotype of structural abnormalities steroid sulphatase deficiency (STS gene)

22q11 duplication syndrome

\begin{tabular}{ll}
\hline Case identified by CNV-seq & Case identified by karyotyping \\
4 & 0 \\
9 & 0
\end{tabular}


22q11 deletion syndrome (DiGeorge syndrome) $\quad 7$

7q11.23 duplication syndrome 3

2q37 monosomy syndrome

Cri du Chat Syndrome (5p deletion)

Williams-Beuren Syndrome (WBS)

$8 \mathrm{p} 23.1$ deletion syndrome

$7 \mathrm{q}$ terminal deletion syndrome

$\mathrm{AZFa}, \mathrm{AZFb}, \mathrm{AZFb}+\mathrm{AZF}$, AZFc syndrome 1

Xq28 (MECP2) microduplication syndrome 1

Wolf-Hirschhorn Syndrome

1q21.1 recurrent microdeletion syndrome

$15 q 26.3$ deletion syndrome

12p13.33 Microdeletion Syndrome

22q11.2 distal deletion syndrome

$8 \mathrm{p} 23.1$ duplication syndrome

15q11q13 duplication syndrome

9q subtelomeric deletion syndrome

RCAD (renal cysts and diabetes) syndrome

Smith-Magenis syndrome

Other(non-syndromic or non-monogenic disease)

total 